\title{
UL'YANOV-TYPE INEQUALITIES AND EMBEDDINGS BETWEEN BESOV SPACES: THE CASE OF PARAMETERS WITH LIMIT VALUES
}

\section{OSCAR DOMÍNGUEZ}

Abstract. In this paper we obtain some limit cases of inequalities of Ul'yanov-type for modulus of smoothness between Lorentz-Zygmund spaces on $\mathbb{T}^{n}$. Corresponding embedding theorems for the Besov spaces are investigated.

Mathematics subject classification (2010): 41A17, 46E30, 46E35, 46M35.

Keywords and phrases: Modulus of smoothness, $K$-functionals, Ul'yanov-type inequalities, generalized Lorentz-Zygmund spaces, Besov spaces, embedding theorems.

\section{REFERENCES}

[1] C. Bennett And K. Rudnick, On Lorentz-Zygmund spaces, Dissertationes Math. 175 (1980), 172.

[2] C. Bennett And R. Sharpley, Interpolation of Operators, Academic Press, Boston, 1988.

[3] J. Bergh AND J. LÖfSTRÖM, Interpolation Spaces. An Introduction, Springer, Berlin, 1976.

[4] H. BRÉZIS AND S. WAINger, A Note on limiting cases of Sobolev embeddings and convolution inequalities, Commun. Partial Differ. Equ. 5 (1980), 773-789.

[5] P. L. Butzer, H. Dyckhoff, E. Görlich And R. L. Stens, Best trigonometric approximation, fractional order derivatives and Lipschitz classes, Canad. J. Math. 29 (1977), 781-793.

[6] A. M. Caetano, A. Gogatishvili And B. Opic, Embeddings and the growth envelope of Besov spaces involving only slowly varying smoothness, J. Approx. Theory 163 (2011), 1373-1399.

[7] F. Cobos AND O. Domínguez, Embeddings of Besov spaces of logarithmic smoothness, Studia Math. 223 (2014), 193-204.

[8] F. Cobos And O. Domínguez, Approximation spaces, limiting interpolation and Besov spaces, J. Approx. Theory 189 (2015), 43-66.

[9] F. Cobos AND O. DomíngueZ, On Besov spaces of logarithmic smoothness and Lipschitz spaces, J. Math. Anal. Appl. 425 (2015), 71-84.

[10] F. Cobos And O. DomíngueZ, On the relationship between two kinds of Besov spaces with smoothness near zero and some other applications of limiting interpolation, J. Fourier Anal. Appl. 22 (2016), $1174-1191$.

[11] F. Cobos, L. M. FernándeZ-Cabrera, T. KÜHn And T. Ullrich, On an extreme class of real interpolation spaces, J. Funct. Anal. 256 (2009), 2321-2366.

[12] F. COBOS AND T. KÜHN, Equivalence of $K$ - and $J$-methods for limiting real interpolation spaces, $J$. Funct. Anal. 261 (2011), 3696-3722.

[13] R. A. DeVore and G. G. Lorentz, Constructive Approximation, Springer, Berlin, 1993.

[14] R. A. DeVore, S. D. Riemenschneider And R. C. Sharpley, Weak interpolation in Banach spaces, J. Funct. Anal. 33 (1979), 58-94.

[15] Z. DitZIAN, Some remarks on approximation theorems on various Banach spaces, J. Math. Anal. Appl. 77 (1980), 567-576.

[16] Z. DitZIAN, Rearrangement invariance and relations among measures of smoothness, Acta Math. Hung. 135 (2012), 270-285.

[17] Z. Ditzian And A. Prymak, Nikol'skii inequalities for Lorentz spaces, Rocky Mountain J. Math. 40 (2010), 209-223. 
[18] Z. Ditzian And S. Tikhonov, Ul'yanov and Nikol'skii-type inequalities, J. Approx. Theory $\mathbf{1 3 3}$ (2005), 100-133.

[19] D. E. Edmunds And W. D. Evans, Hardy Operators, Function Spaces and Embeddings, Springer, Berlin, 2004.

[20] D. E. Edmunds, P. GURKa AND B. OPIC, Double exponential integrability of convolution operators in generalized Lorentz-Zygmund spaces, Indiana Univ. Math. J. 44 (1995), 19-43.

[21] D. E. Edmunds, P. GURKa AND B. OpIC, Double exponential integrability, Bessel potentials and embedding theorems, Studia Math. 115 (1995), 151-181.

[22] D. E. EdmundS AND B. OPIC, Limiting variants of Krasnosel'skii's compact interpolation theorem, J. Funct. Anal. 266 (2014), 3265-3285.

[23] W. D. Evans AND B. OpIC, Real interpolation with logarithmic functors and reiteration, Canad. J. Math. 52 (2000), 920-960.

[24] W. D. Evans, B. OPIC AND L. PICK, Interpolation of operators on scales of generalized LorentzZygmund spaces, Math. Nachr. 182 (1996), 127-181.

[25] W. D. Evans, B. OPIC AND L. PICK, Real interpolation with logarithmic functors, J. Inequal. Appl. 7 (2002), 187-269.

[26] A. Gogatishvili, B. Opic, S. Tikhonov and W. Trebels, Ulyanov-type inequalities between Lorentz-Zygmund spaces, J. Fourier Anal. Appl. 20 (2014), 1020-1049.

[27] A. GogatishVILI, B. OpIC AND W. TREBELS, Limiting reiteration for real interpolation with slowly varying functions, Math. Nachr. 278 (2005), 86-107.

[28] A. Gogatishvili, L. Pick AND J. SCHNEIDER, Characterization of a rearrangement-invariant hull of a Besov space via interpolation, Rev. Mat. Complut. 25 (2012), 267-283.

[29] M. L. Gol'dman, Embedding of constructive and structural Lipschitz spaces in symmetric spaces, Proc. Steklov Inst. Math. 173 (1987), 93-118.

[30] J. Gustavsson, A function parameter in connection with interpolation of Banach spaces, Math. Scand. 42 (1978), 289-305.

[31] D. D. Haroske AND H. TRiebel, Embeddings of function spaces: a criterion in terms of differences, Complex Var. Elliptic Equ. 56 (2011), 931-944.

[32] V. I. KolyadA, Rearrangements of functions and embedding theorems, Russian Math. Surveys 44 (1989), 73-117.

[33] E. Nursultanov And S. Tikhonov, A sharp Remez inequality for trigonometric polynomials, Constructive Approx. 38 (2013), 101-132.

[34] H.-J. SchmeISSER AND H. TRIEBel, Topics in Fourier Analysis and Function Spaces, Wiley, Chichester, 1987.

[35] B. Simonov And S. TiKhonov, Sharp Ul'yanov-type inequalities using fractional smoothness, J. Approx. Theory 162 (2010), 1654-1684.

[36] E. M. Stein ANd G. Weiss, Introduction to Fourier Analysis on Euclidean Spaces, Princeton Univ. Press, Princeton, 1971.

[37] S. TikHonov, On modulus of smoothness of fractional order, Real Anal. Exchange 30 (2004/2005), $507-518$.

[38] W. Trebels, Inequalities for moduli of smoothness versus embeddings of function spaces, Arch. Math. (Basel) 94 (2010), 155-164.

[39] H. Triebel, Interpolation Theory, Function Spaces, Differential Operators, North-Holland, Amsterdam, 1978.

[40] P. L. UL'YANOv, The imbedding of certain function classes $H_{p}^{\omega}$, Math. USSR-Izv. 2 (1968), 601-637.

[41] U. WestPhal, An approach to fractional powers of operators via fractional differences, Proc. Lond. Math. Soc. III (Ser. 29) (1974), 557-576. 